\title{
FINAL POSITIVITY CORRECTION IN THE HARMONIC METHOD
}

\author{
M. DAHMS \\ Institut für Werkstofforschung, GKSS-Forschungszentrum, Max-Planck-Straße, \\ D-2054 Geesthacht
}

(17 May, 1992)

Final positivity correction of an ODF containing negative densities can be carried out using a linear variant of the exponential method. The phone-concept is automatically included. The final positivity correction corrects the truncation error of the harmonic method if the given series coefficients describe a totally positive axis distribution function.

KEY WORDS Final positivity correction, exponential method, truncation error, phone, isotropic texture component.

\section{INTRODUCTION}

Since the beginning of the harmonic method one of its major drawbacks was the occurrence of negative values in the ODF and in recalculated pole figures because of incompatibilities of the measured pole figures, "ghost" effects and series truncation (Bunge, 1982). Negative values are intrinsically possible in Fourier series although they are physically impossible for density functions. Even the positivity method for even- and odd-order coefficients (Dahms \& Bunge, 1989) cannot totally exclude negative densities (Dahms, 1992a). Although the introduction of the "phone"-concept (Matthies, 1984) into pole figure inversion lead to some improvement, negative densities may still be present in the ODF (Dahms, 1992b). Here, intrinsically positive series expansion using a quadratic (Van Houtte, 1983) or an exponential (Van Houtte, 1991) form can lead to an absolute positive ODF. If such an ODF is described by a finite Fourier series, only truncation errors can lead to negative values in the recalculated ODF. Wagner's final correction (1992) also results in a positive ODF, but it is in fact a simplification of the first approximation of the complete ODF in the exponential method as will be shown later.

\section{MATHEMATICAL CONSIDERATIONS}

An orientation distribution function (ODF) of a polycrystalline aggregate $f(g)$ can be described by series expansion using symmetrized general spherical harmonic functions $\ddot{T}_{l}^{\mu \nu}(g)$ with coefficients $C_{l}^{\mu \nu}$ (Bunge, 1982)

$$
f(g)=\sum_{l=0}^{\infty} \sum_{\mu=1}^{M(l)} \sum_{v=1}^{N(l)} C_{l}^{\mu v} \dot{\tilde{T}}_{l}^{\mu v}(g)
$$


where the variable $g$ represents the crystal orientation, e.g. described by the eulerian angles $\varphi_{1}, \Phi, \varphi_{2}$, and $M(l)$ and $N(l)$ are integers defined by crystal and sample symmetries, respectively.

In practice, series expansion can only be carried out up to a maximum index $l_{\max }$, the degree of series expansion $L$. In the case of measured orientation distributions (pole figures or individual orientation measurements), this is due to the limited number of data points. In general and also for theoretical orientation distributions, the absolute limit is defined by the computer capacity. Equation (1) is then transformed to

$$
f(g)=\sum_{l=0}^{L} \sum_{\mu=1}^{M(l)} \sum_{v=1}^{N(l)} C_{l}^{\mu v} \dot{\bar{T}}_{l}^{\mu v}(g)+A_{L}
$$

where $A_{L}$ is the truncation error (Bunge, 1982).

The ODF may be split in an even and an odd part $\tilde{f}(g)$ and $\tilde{\tilde{f}}(g)$, respectively.

$$
f(g)=\tilde{f}(g)+\tilde{f}(g)
$$

and

$$
\tilde{f}(g)=\sum_{l=1(2)}^{\infty} \sum_{\mu=1}^{M(l)} \sum_{v=1}^{N(l)} C_{l}^{\mu v} \dot{\hat{T}}_{l}^{\mu v}(g)
$$

In series terms, it is written

$$
\tilde{f}(g)=\sum_{l=1(2)}^{\infty} \sum_{\mu=1}^{M(l)} \sum_{v=1}^{N(l)} C_{l}^{\mu v} \dot{\bar{T}}_{l}^{\mu v}(g)
$$

In principal, the even part can be directly determined from measured pole figures by pole figure inversion up to a certain degree $L$ whereas the calculation of the odd part needs the additional information that the ODF is a non-negative function.

$$
f(g) \geq 0
$$

Also the odd part can only be determined up to a certain degree, e.g. $L-1$. If we define

and

$$
\tilde{f}^{L}(g)=\sum_{l=0(2)}^{L} \sum_{\mu=1}^{M(l)} \sum_{v=1}^{N(l)} C_{l}^{\mu v} \dot{\tilde{T}}_{l}^{\mu v}(g)
$$

it is

$$
\tilde{\tilde{f}}^{L}(g)=\sum_{l=1(2)}^{L-1} \sum_{\mu=1}^{M(l)} \sum_{v=1}^{N(l)} C_{l}^{\mu v} \dot{\bar{T}}_{l}^{\mu v}(g)
$$

$$
f(g)=\tilde{f}^{L}(g)+\tilde{f}^{L}(g)+A_{L}
$$

Because measured data are generally containing errors and series expansion is finite in reality, the "true" ODF is only approximated by the ODF $f_{0}(g)$ calculated from experimental data. It is

$$
f(g)=f_{0}(g)+\Delta f(g)
$$

where the $\Delta$-term includes experimental, numerical and truncation errors as well as the "indeterminability" error of the odd part, see e.g. (Matthies, 1984). In the 
harmonic method, $f_{0}(g)$ is calculated by least squares methods (Bunge, 1982; Dahms, Bunge, 1989). Then, it is possible that $f_{0}(g)$ contains negative regions, because a Fourier series like Eq. (1) is not intrinsically positive.

It is impossible to determine the residual function $\Delta f(g)$ exactly. Only that part can be calculated which transforms the partially negative function $f_{0}(g)$ into a non-negative function. The limiting constraint is the requirement that the correction function $\Delta \tilde{f}(g)$ should be minimized, with

$$
\Delta \tilde{f}(g)=\tilde{f}^{L}(g)-\tilde{f}_{0}(g)
$$

The problem now arises, how to find a positive ODF from a given ODF containing negative values where both ODF's should have the same even part $\tilde{f}^{L}(g)$ or at least a minimum difference.

A positive approximation of an ODF containing negative values must be defined. In the exponential method, this is (VH refers to Van Houtte (1991)):

$$
f_{1}^{\prime}(g)= \begin{cases}f_{0}(g), & \text { for } f_{0}(g) \geq r \\ r \exp \left(f_{0}(g)-r\right), & \text { for } f_{0}(g)<r\end{cases}
$$

Instead of $r \exp \left(f_{0}(g)-r\right)$, any other reasonable assumption can be made such as the definition of a random texture component.

$$
f_{1}^{\prime}(g)=r, \text { for } f_{0}(g)<r
$$

For $r=0$, Eq. (12) and Eq. (12') are identical.

We shall now switch to the generalized index form because we have entered the iteration loop. Renormalization is achieved by

$$
k_{n}^{\prime}=\oint f_{n}^{\prime}(g) d g \quad(\mathrm{VH} 25)
$$

and then

$$
f_{n}(g)=f_{n}^{\prime}(g) / k_{n}^{\prime} \quad-(\mathrm{VH} 26)
$$

If the procedure is terminated at $n=1$, and if $r=0$ is used in Eq. (12) for the approximation of the complete ODF, exactly Wagner's (1992) final correction is realized.

The normalized function $f_{n}$ is developed into a series by

$$
C_{l}^{\mu v}(n)=(2 l+1) \oint \dot{T}_{l}^{\mu v}(g) f_{n}(g) d g
$$

being used to calculate the even part $\tilde{f}_{n}^{L}(g)$ of the estimated ODF $f_{n}(g)$. We now compare $\tilde{f}_{n}^{L}(g)$ with the original "even" ODF $\tilde{f}_{0}(g)$ using Eq. (11), having to decide whether the solution, Eq. (14), is sufficient or not:

$$
\begin{gathered}
\min \left|\Delta \tilde{f}_{n}(g)\right|<a \\
\oint \Delta \tilde{f}_{n}(g)^{2} d g<b \quad(\mathrm{VH} 17) \\
\oint f_{n}^{\prime}(g) d g-1<c
\end{gathered}
$$

where $a, b, c$ are constants to be defined by the user. If the solution is 
insufficient, a correction of $f_{n}(g)$ is obtained by

$$
f_{n+1}^{\prime}(g)=f_{n}(g) \exp \frac{\Delta \tilde{f}_{n}(g)}{f_{n}(g)}
$$

Here, we can see the major advantage of the exponential method because during the iteration, each estimate of $f(g)$ is positive. For brevity, we define

$$
\delta f_{n}(g)=\frac{\Delta \tilde{f}_{n}(g)}{f_{n}(g)}
$$

For $\delta f_{n}(g) \gg 1$, Eq. (17) diverges, i.e. very large values may occur for $f_{n+1}^{\prime}(g)$ because of the exponential function in Eq. (18). Van Houtte (1992) has tackled this problem by modifying the basic function. Unfortunately, this disturbs the mathematical clarity of his solution. Additionally, for $\delta f_{n}(g) \ll 0, f_{n+1}^{\prime}(g)$ and hence also $f_{n+1}(g)$ approaches zero. Then Eq. (17) diverges in the next iteration step. For $\left|\delta f_{n}(g)\right| \ll 1$, Eq. (17) results in

$$
f_{n+1}^{\prime}(g)=f_{n}(g)+\Delta \tilde{f}_{n}(g)=\tilde{f}_{0}(g)+\tilde{f}_{n}(g)
$$

which is the final solution $f_{N}(g)$. We can see here that $\left|\delta f_{n}(g)\right| \ll 1$, results in unchanged even order coefficients because $\tilde{f}_{0}(g)$ is kept. Equation (19) can also be used instead of Eq. (17), for $\left|\delta f_{n}(g)\right| \nless k 1$, but then, the simple positivity condition must be introduced additionally:

$$
f_{n+1}^{\prime}(g)= \begin{cases}f_{n}(g)+\Delta \tilde{f}_{n}(g), & \text { for } f_{n}(g)+\Delta \tilde{f}_{n}(g) \geq r \\ r, & \text { for } f_{n}(g)+\Delta \tilde{f}_{n}(g)<r\end{cases}
$$

The final solution $f_{N}(g)$ does contain harmonic terms of infinite order. If $f_{N}(g)$ is developed into a finite series using Eq. (14) and the ODF is then recalculated, it may contain negative values again-the truncation error. Because of that, we suggest to use $f_{N}(g)$ for graphic representation.

A major variant should be discussed here additionally. The difference $\Delta \tilde{f}_{n}(g)$ can be generalized. It can be understood as a difference between a general known part of the ODF and its approximation-which should be in fact zero. If just a few even order coefficients are known with high precision, e.g. if only a limited number of pole figures was measured, the degree of series expansion for the final positivity correction can be used. This leads to a speed-up of the whole iteration loop which is very important in the case of low symmetries. This procedure implies the description of the ODF by a limited number of strong coefficients which is similar to the description by a finite number of components (Lücke et al., 1981; Helming \& Eschner, 1990). It has to be checked separately whether this reduction leads to falsification of the ODF.

\section{EXAMPLE}

The effect of final positive correction will be checked by a real quartz-texture (Braun et al., 1991). The choice of a real texture is important because only here, considerable high order coefficients can be found. In Figure 1, the mean absolute $C$-coefficients are shown up to the degree of series expansion $L=23$ as they were 


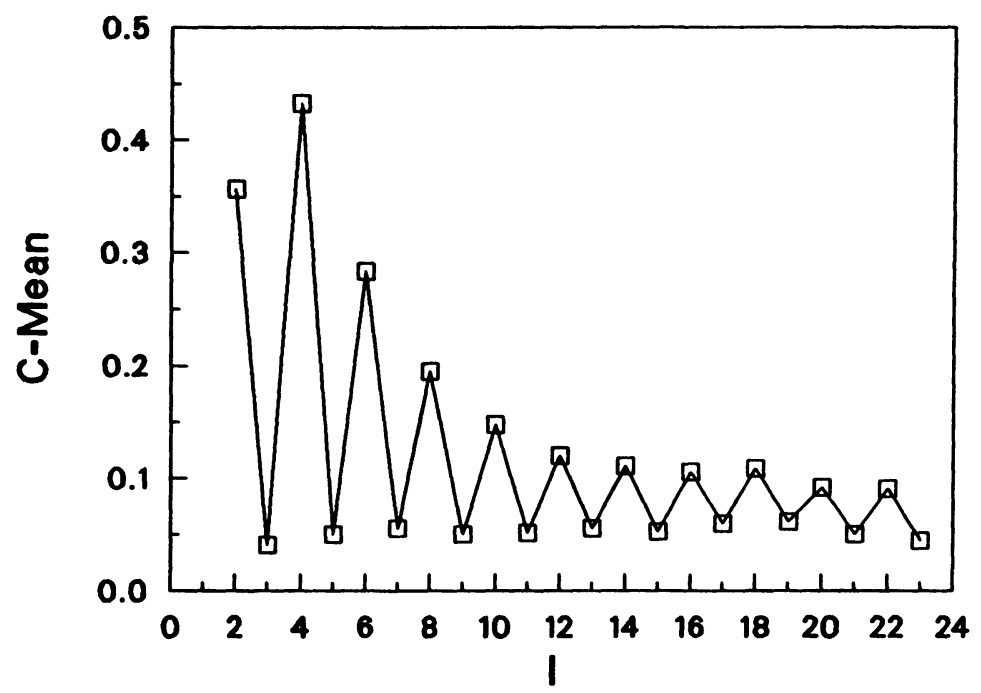

Figure 1 Mean absolute $C$-coefficients vs. degree of series expansion $l$ for a quartz-texture.

calculated using the positivity method. For the even-order coefficients, a phone of 0.5 was introduced as described in (Dahms, 1992). This leads to a minimum value in the general axis distribution function of 0.11 . For the odd-order coefficients, a phone of 0.1 was introduced as described in (Dahms and Bunge, 1989). It was impossible to obtain a fully positive complete ODF. The resulting minimum value was -0.52 .

As a first step, the precision of the numerical integration, Eq. (14), was checked by calculating

$$
\Delta C=\sum_{l, \mu, v} \frac{1}{2 l+1}\left(C_{l}^{\mu v}(0)-C_{l}^{\mu v}(1)\right)^{2}
$$

for even $l$ and $r=-0.6$ up to $L=22$. This term is equivalent to Eq. (16b). In the case of perfect numerical integration, $\Delta C$ should be zero. The integration was carried out in steps of $2.5^{\circ}$ in $\varphi_{1}$ and $\Phi$ and $7.5^{\circ}$ in $2 \sigma$, respectively. This is the unusual matrix for graphical representation. The resulting value was $\Delta C=$ $6.7 \cdot 10^{-6}$ which is small compared with expected errors in the range of $10^{-2} \ldots 10^{-4}$ according to Van Houtte (1991). For $L=10$, the error was reduced to $\Delta C=1.6 \cdot 10^{-6}$.

The iterative procedure described above was now applied using the isotropic component of the axis distribution function $r=0.11$. In Figure 2 , the decrease of $\Delta C$ during the iterative procedure can be seen. For $L=10$, it quickly tends to the numerical error, i.e. the even-order coefficients of low order are nearly kept during the final positivity correction. For $L=22$, the reduction of $\Delta C$ is less pronounced. The iteration was stopped after 3000 CPU sec.

As said before, the final positive ODF contains series terms of infinite order. In order to check their influence, the final positive ODF with $L=22$ during the iteration was developed into a series and recalculated up to $L=23$. The minimum 


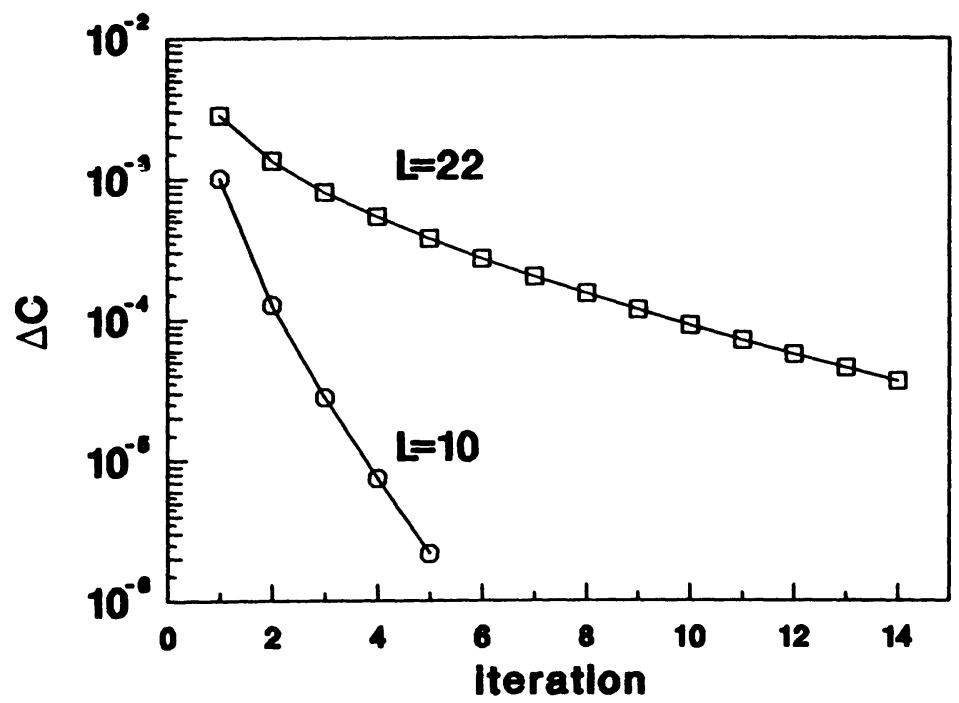

Figure 2 Decrease of the error $\Delta C$ during the iterative final positivity correction for $L=10$ and $L=22$.

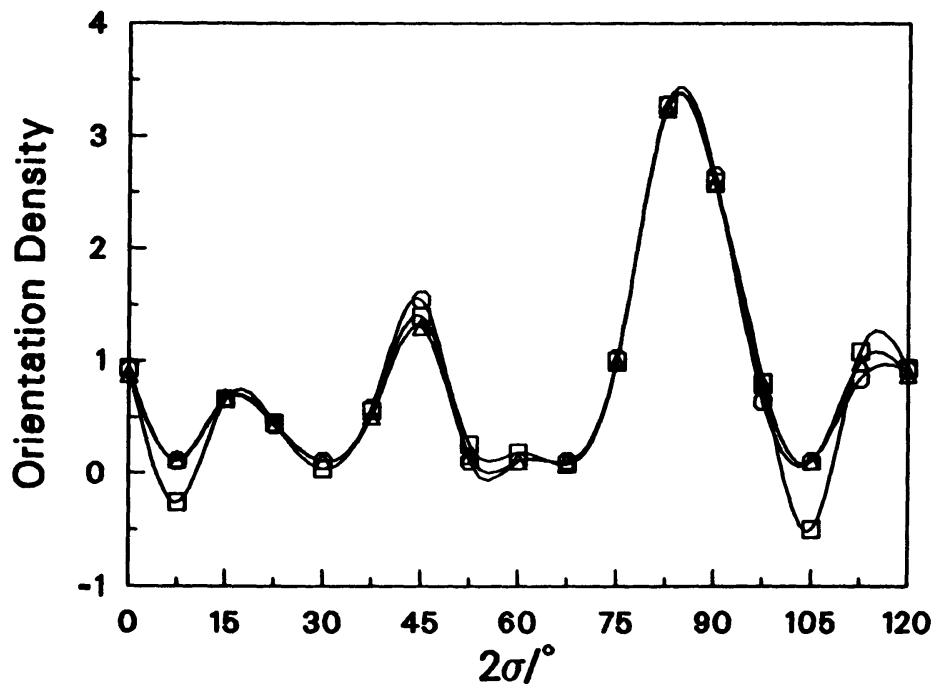

Figure 3 Section $\varphi_{1}=25^{\circ}, \Phi=50^{\circ}$ of the quartz ODF determined by the positivity, method ( $\square$ ), final positivity correction using $L=10(\triangle)$ and $L=22(O)$, respectively. 


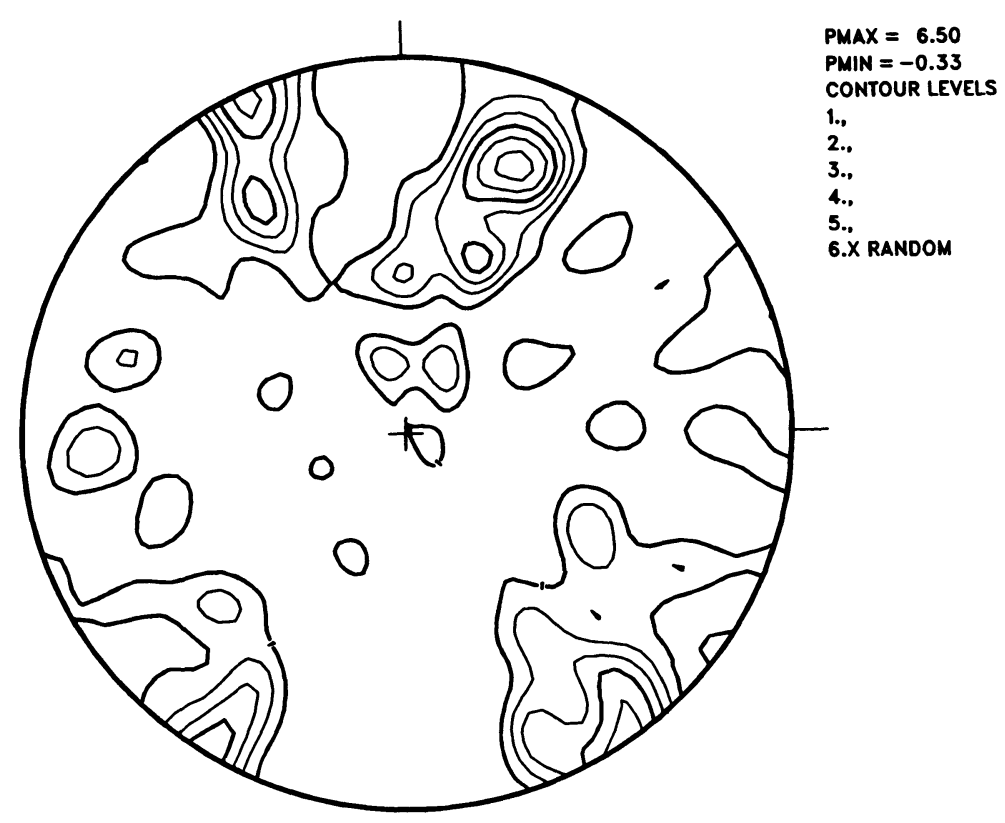

a)

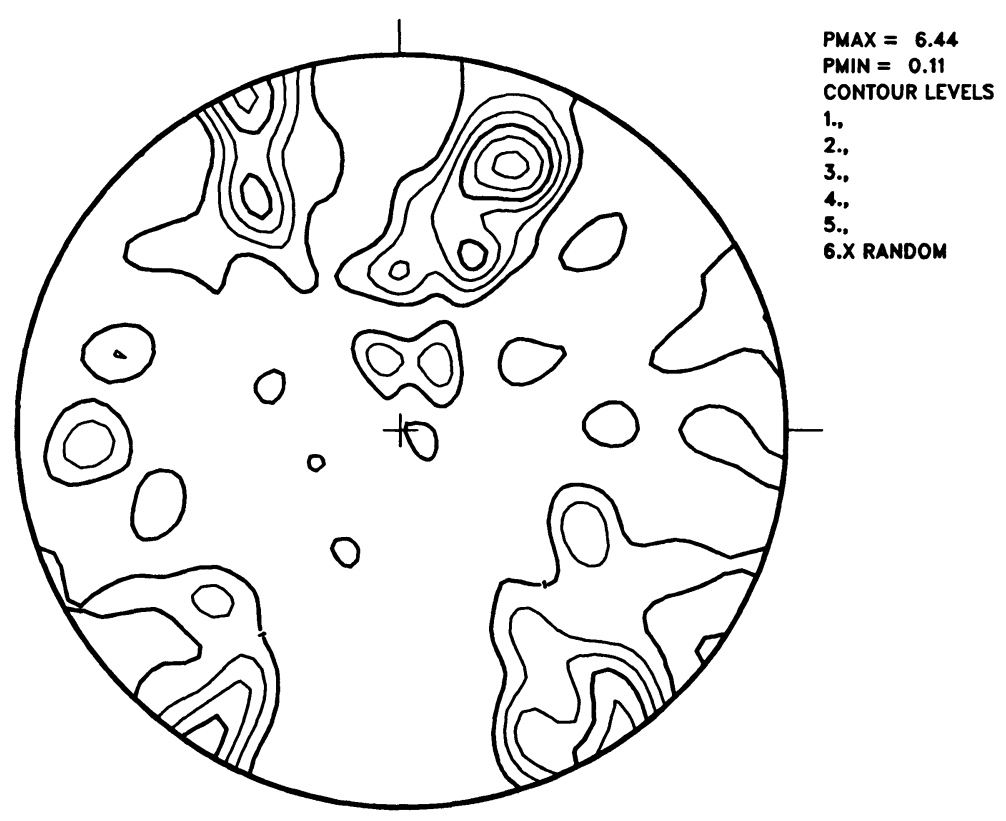

b)

Figure 4 Section $2 \sigma=0^{\circ}$ of the quartz ODF determined by a) the positivity method, b) final positivity correction using $L=10$ and c) final positivity correction using $L=22$. 


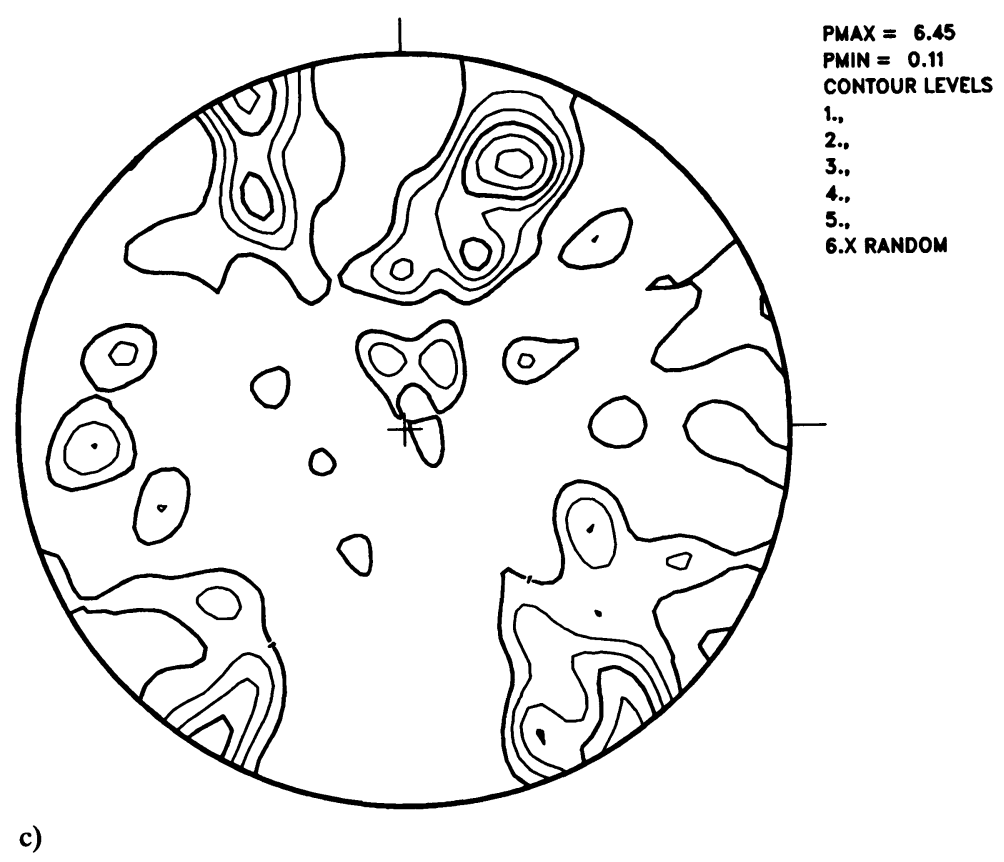

Figure 4 (continued)

value of the ODF was -0.55 . This shows that the negative residuum at the end of the positivity method is only due to the series truncation error.

In order to show that the final positivity correction does not change the ODF remarkably and that also the reduction of the degree of series expansion during iteration is uncritical, the section $\varphi_{1}=25^{\circ}, \Phi=50^{\circ}$ of all ODFs is shown in Figure 3. This section was chosen because here, the minimum value was found in the positivity method. It can be seen that the final positivity correction raises the minimum value to the assumed phone, and the other data points are just slightly adjusted in order to keep the even-order coefficients constant up to the given degree $L$. No significant change in the texture-peaks takes place. This can also be seen in Figure $4 a-c$, where the sections $2 \sigma=0^{\circ}$ of all ODFs are shown.

\section{CONCLUSIONS}

Final positivity correction of an ODF containing negative densities is easily possible using a linear approach including the phone-concept. Reducing the degree of series expansion for the iteration is uncritical. If the axis distribution function is totally positive, final positivity correction only corrects the truncation error. Final positivity correction is only necessary, if the representation of a principally positive ODF without truncation error is desired. 


\section{References}

Braun, G., Siegesmund, S. and Dahms, M. (1991). The influence of quartz textures on the seismic anisotropy in lower crystal granulites. Journal of Structural Geology 13, 955-966.

Bunge, H. J. (1982). Texture Analysis in Materials Science Butterworth, London.

Dahms, M. and Bunge, H. J. (1989). The iterative series expansion method for quatitative texture analysis_part I: general outline. J. Appl. Cryst. 22, 439-447.

Dahms, M. (1992a). The iterative series expansion method for quantitative texture analysis, part II: applications. J. Appl. Cryst. 25, 258-267.

Dahms, M. (1992b). Introduction of the phone-concept into pole figure inversion using the iterative series expansion method. Textures and Microstructures 19, 169-174.

Helming, K. and Eschner, T. (1990). A new approach to texture analysis of multiphase materials using a texture component model. Crys. Res. Technol. 25, K203-K208.

Lücke, K., Pospiech, J., Virnich, K. H. and Jura, J. (1981). On the problem of reproduction of the true orientation distribution from pole figures. Acta Met. 29, 167-186.

Matthies, S. (1984). The present situation in the field of ODF reproduction and ghost correction. In ICOTOM 7, Proc. 7th Intern. Conf. on Textures of Materials, edited by C. M. Brakman, P. Jongenburger and E. J. Mittenseijer, pp. 737-751, Netherlands Society for Materials Science: Noordwijkerhout.

Van Houtte, P. (1983). The use of a quadratic form for the determination of non-negative texture functions. Textures and Microstructures 6, 1-20.

Van Houtte, P. (1991). A method for the generation of various ghost correction algorithms-the example of the positivity method and the exponential method. Textures and Microstructures 13, 199-212.

Wagner, F. (1992). Determination of fully positive O.D.F.s in the frame of the harmonic method. Textures and Microstructures 19, 59-61. 